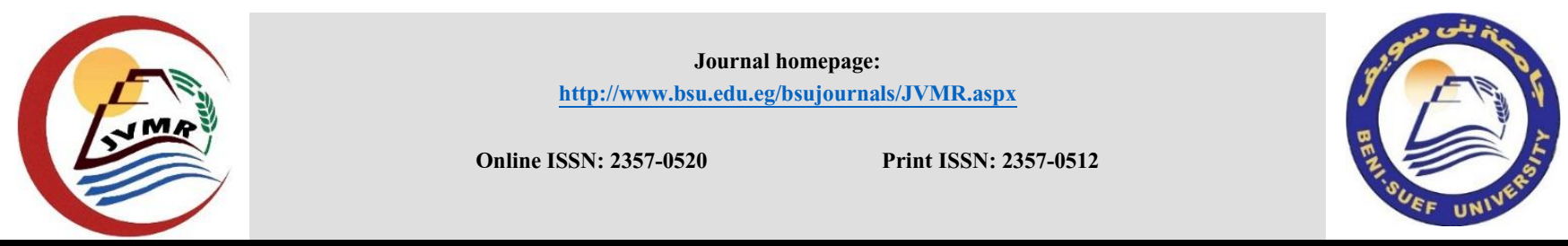

Original Research Article

\title{
Serogrouping and resistance gene detection in avian pathogenic E.coli isolated from broiler chickens
}

\author{
Asmaa Abd Elatiff ${ }^{1}$, Azza A. El-Sawah ${ }^{2}$ Mohamed M. Amer ${ }^{3}$, Al-Hussein M. Dahshan ${ }^{2}$, Hala Salam ${ }^{4}$, Salama A.S.Shany ${ }^{2}$ \\ ${ }^{1}$ Veterinarian, Faculty of agriculture, El Minya University. \\ ${ }^{2}$ Poultry Diseases, Faculty of Veterinary Medicine, Beni-Suef University, Beni-Suef 62511, Egypt. \\ ${ }^{3}$ Poultry Diseases, Faculty of Veterinary Medicine, Cairo University, Giza12618, Egypt \\ ${ }^{4}$ Bacteriology, Mycology and Immunology, Faculty of Veterinary Medicine, Beni-Suef University.
}

\begin{abstract}
E. coli infection in poultry is one of the principle causes of mortality and morbidity in chickens and turkeys resulting in raardation of growth and decreased feed conversion rate. The most common form of collibacillosis is characterized as an initial respiratory infection (air sacculitis) followed by generalized septicemia, perihepatitis and pericarditis. The present study aimed to identify the bacteria associated with pericarditis , air sacculitis and perihapatitis in Egyptian broilerchickens. A total 300 samples of diseased and dead broilers from 3-6 weeks age were collected. The isolated bacteria included E. coli, Klebsiella spp., Shigella spp. And Enterobacter spp. Sero-grouping of the isolated E. coli strains revealed O125, O158, O55,O129, O20, O6 , O8 , O27, O115, O142 and un-typed strains with prevalence of $16 \%, 12 \%, 8 \%, 8 \%, 8 \%, 8 \%, 8 \%, 8 \%, 4 \%, 4 \%$ and $16 \%$ respectively. The majority of $E$. coli isolates were sensitive to colistin sulphate (38\%) and Norofloxacin(38\%) followed by ciprofloxacin (19\%), cefotaxim (19\%) and Ofloxacin (19\%). On the other hand, E. coli were resistant (100\%)to amoxicillin, clindamycin , Erythromycin and streptomycin. PCR analysis for antibiotic resistance genes of E. coli detected that 12 serogroups isolates were positive using the specific primers for Aada2, BlaCTX and TetA(A) genes. The current study demonstrated the high prevalence of $E$. coli indeed broilers suffered from pericarditis, perihepatitis and airsacculitis . Measures are needed to control E. coli contamination in poultry farms to reduce economic losses caused by infection of E.coli.
\end{abstract}

\begin{tabular}{l} 
ARTICLE INFO \\
\hline Article history: \\
Received: $3 / 2019$ \\
Accepted $4 / 2019$ \\
Online $4 / 2019$ \\
\hline Keywords: \\
Chickens \\
Broilers \\
E. coli \\
Pericarditis \\
FCR
\end{tabular}

\section{Introduction}

E. coli is a member of the family Enterobacteriaceae, which may constitute a great hazard to poultry industry causing high mortality, loss of weight and reduction of egg production (Bandyopadhay and Dhawedkar, 1984). E. coli infection is one of the serious problems that cause a great threat to the profitability of birds' enterprises allover the word. The most common form of collibacillosis is characterized as an initial respiratory infection (air sacculitis) in 3-12 weeks old broiler chickens and turkeys, which is frequently followed by generalized septicemia, perihepatitis and pericarditis.
Vandekerchove et al. (2004 a) observed outbreaks of acute mortality in layer flocks in Europe due to colisepticemia. They described the disease and identified the serotypes of the APEC present in these outbreaks. Salama et al., (2007) recovered 5 serogroups of $\mathrm{E}$. coli identified as $\mathrm{O} 1, \mathrm{O} 2, \mathrm{O} 6, \mathrm{O} 78$ and $\mathrm{O} 126$. Out of 33 isolates derived from a total of 60 samples collected from colisepticemic chickens isolated on nutrient agar and MacConkey agar and colonies examined for their colonial morphology, microscopically and biochemically using API 20E identification system. El-Jakee et al., (2012) identified 28 isolates recovered from cloacal swabs of diarrheic chicken; they were O2, O6, O8, O26, O27, O78, O86, O111, O128, 
O136 and O157. Sarah et al ., (2015): recovered E coli serovars from different sources of poultry broiler farms which were; untypable $\mathrm{E}$. coli isolates followed by $\mathrm{O} 26$; then $\mathrm{O} 2$, O124, O125, and O115. Food animals and their production environments are source and reservoirs of both resistant bacteria and resistance genes that could be transferred to.

E. coli bacteria often carry multiple drug resistance plasmids and mixing of species in the intestines allows $E$. coli to accept and transfer plasmids from one to other bacteria, [salyers et al 2004]. Many authors had clarified a lot about the antibiotic resistance profile of bacteria including E. coli in poultry farms in Egypt [Yousef et al 2013 - Moawad et al 2017] found that 87 APEC isolates from septicaemic broilers at the molecular level showed multidrug-resistant phenotypes, particularly against ampicillin, kanamycin, ciprofloxacin, levofloxacin, streptomycin, gentamycin, ofloxacin, norfloxacin, and ceftriaxone. Hering et al 2016; Suggested that the analysis of fecal samples in sufficient to determine cefotaxim-resistant $E$. coli in broiler farm but that cefotaxime resistance is a good proxy for the presence of ESBL- or plasmidic AmpC-betalactamases and the prevalence of broiler farms with cefotaxime-resistant E. coli in Germany is very high. Nawaz et al (2016) studied that the efficacy of two probiotics of different origins (yeast and bacterial based) on the growth performance, immune response, carcass characteristics and nutrient digestibility of broilers.

The aim of the present study was to isolate and identify the bacteria associated with mortality and poor feed conversion from broiler chickens

\section{Materials and methods}

2.1 Case history: Recently dead or diseased broilers (34weeks) were collected from farms in Elminya Province suffer from pericarditis, perihepatitis and air sacculitis .

\subsection{Chickens Samples}

A total of 300 samples were collected from different organs (liver , heart and lung) were transferred immediately to sterile buffered peptone water, then wrapped with ice, kept in box and transferred to the laboratory to be submitted to bacteriological examination .

2.3. Detection of $\mathbf{E}$, coli by conventional method: it was done according to Quinn et al. (2002)

\subsubsection{Selective enrichment of $E$. coli}

For bacteriological examination $0.5 \mathrm{gm}$ of liver, heart and lung were inoculated into tryptone soya broth. All inoculated media were incubated aerobically at $37^{\circ} \mathrm{C}$ for $16-18 \mathrm{hrs}$.
2.3.2. Colonization of $E$. coli on selective differential solid media

Loopfuls from the inoculated broth were streaked onto SSagar, XLD agar, MacConkey agar and EMB agar (Cheesbrough, 2000) Suspected E. coli colonies were purified and kept for further morphological and cultural identification.

\subsubsection{Microscopic examination}

Gram's stain was prepared and used as described by (Cruickshank et al. 1975) for morphological characters (Koneman et al., 1992; Quinn et al., 2002)).

\subsubsection{Confirmatory API20 E biochemical test}

Analytical profile index 20 E (API 20 E Biochemical rapid tests, Bio-Meraux, France) was used for identification of Enterobacteriaceae. The test was carried out according to the instructions of the manufacturer (Bio Meraux, France). Only pure cultures of a single organism were used.

\subsubsection{Serological identification of E. coli}

Isolated E. coli were serogrouped at the Animal Health Research Institute, Dokki, Giza according to (Koneman et al., 1992). Suspected microorganisms were subjected to serological typing by slide agglutination test using standard polyvalent and monovalent $E$. coli antisera.

\subsection{Virulence testing of isolated bacteria}

\subsubsection{Congo red binding assay}

Selected organisms were tested for virulence using Congo red binding assay and detection of hemolytic activity (Berkhoff and Vinal, 1986). Briefly, isolates were tested for its growth status on Congo red medium after incubation for 24 hours at $35^{\circ} \mathrm{C}$ then left at room temperature for additional two days for better results

\subsubsection{Hemolytic activity on Blood A gar}

The test was conducted according to (Marilda et al., 1990), where overnight cultures of tested organisms were streaked onto Blood agar base containing $10 \%$ defibrinated sheep blood and incubated at $37^{\circ} \mathrm{C}$ for $24 \mathrm{hr}$. Complete hemolysis 
was recognized as $\beta$ - hemolysis while, weak incomplete hemolysis was recognized as $\propto$ - hemolysis.

\subsection{Antibiogram sensitivity test :}

Twenty one serogrouped E. coli were tested by the single-disc diffusion method according to Mary and Usha (2013). Sensitivity discs and Muller-Hinton agar (Oxoid Limited, Basingstoke, Hampshire, UK) . The tested antimicrobials

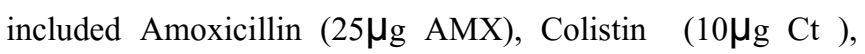
Ciprofloxacin $(5 \mu \mathrm{g} \quad \mathrm{CP}), \quad$ Erythromycin $(15 \mu \mathrm{gE})$, Enerofloxacin $(5 \mu \mathrm{gK})$, Cefotax $(50 \mu \mathrm{g})$, clindamycin $(2 \mu \mathrm{g})$, Ofloxacin $(5 \mu \mathrm{g})$, Norfloxacin $(10 \mu \mathrm{g}$ NOR), Doxycycline (30 $\mu g$ Do), Streptomycin (10 $\mu \mathrm{g}$ S) and Sulphamethoxazol \& trimethoprim $(25 \mu \mathrm{g} \mathrm{SXT})$ table (1). The interpretation of inhibition zones of tested culture was according to NCCLS, 2002).

\section{Table (1): Antimicrobial susceptibility profile of E , coli isolates}

\begin{tabular}{|c|c|c|c|c|c|c|c|}
\hline \multirow{2}{*}{$\begin{array}{l}\text { Antimicrobial } \\
\text { discs }\end{array}$} & \multirow[t]{2}{*}{ conc } & \multicolumn{2}{|c|}{ Sensitive } & \multicolumn{2}{|c|}{$\begin{array}{c}\text { Intermedia } \\
\text { te }\end{array}$} & \multicolumn{2}{|c|}{ Resistant } \\
\hline & & No & $\%$ & No & $\%$ & No & $\%$ \\
\hline Doxycycline & Do30 & 1 & 4.7 & 2 & 9.5 & 18 & 85.7 \\
\hline Ciprofloxacin & Cip5 & 3 & 14.2 & 2 & 9.5 & 16 & 76 \\
\hline Amoxicillin & Ax25 & $\mathbf{0}$ & $\mathbf{0}$ & $\mathbf{0}$ & $\mathbf{0}$ & 21 & 100 \\
\hline Cefotaxime & Ctx30 & 4 & 19 & $\mathbf{0}$ & $\mathbf{0}$ & 17 & 80.9 \\
\hline $\begin{array}{c}\text { Colistin } \\
\text { sulphate }\end{array}$ & Ct10 & 8 & 38 & $\mathbf{0}$ & $\mathbf{0}$ & 13 & 61.9 \\
\hline $\begin{array}{c}\text { Sulphamettho } \\
\text { xazole/trimet } \\
\text { hoprime }\end{array}$ & Sxt25 & 3 & 14.2 & 5 & 23.8 & 13 & 61.9 \\
\hline Clindamycin & Da2 & $\mathbf{0}$ & $\mathbf{0}$ & $\mathbf{0}$ & $\mathbf{0}$ & 21 & 100 \\
\hline $\begin{array}{c}\text { Erythromyci } \\
n\end{array}$ & E15 & 0 & 0 & 0 & 0 & 21 & 100 \\
\hline Streptomycin & S10 & $\mathbf{0}$ & $\mathbf{0}$ & $\mathbf{0}$ & $\mathbf{0}$ & 21 & 100 \\
\hline Ofloxacin & Of $x 5$ & 2 & 19 & 3 & 14.2 & 16 & 76 \\
\hline Norofloxacin & Nor10 & 3 & 14.2 & 6 & 28.5 & 12 & 57 \\
\hline
\end{tabular}

\subsection{Detection of antibiotic resistance gene}

Twelve E.coli were tested for presence or absences of resistance genes mainly resistance to streptomycin (Aada 2), tetracycline (TetA (A) and cefotaxime ( Bla CTX) were determined by PCR and the set of primers used for each gene is shown in Table 2 at the Animal Health Research Institute, Dokki, Giza.

\section{Table(2). Gene primer sequence}

\begin{tabular}{|c|c|c|c|}
\hline Gene & $\begin{array}{c}\text { Primer Sequence } \\
5^{\prime}-3^{\prime}\end{array}$ & $\begin{array}{r}\text { Amplified } \\
\text { product }\end{array}$ & References \\
\hline $\operatorname{Tet} A(A)$ & $\begin{array}{l}\text { GGTTCACTCGAACGACGTCA } \\
\text { CTGTCCGACAAGTTGCATGA }\end{array}$ & 576bp & $\begin{array}{l}\text { Randall et al. } \\
2004\end{array}$ \\
\hline AadA2 & $\begin{array}{l}\text { TGTTGGTTACTGTGGCCGTA } \\
\text { GATCTCGCCTTTCACAAAGC }\end{array}$ & 622 bp & $\begin{array}{c}\text { Walker et al. } \\
(2001)\end{array}$ \\
\hline Bla $_{C T X}$ & $\begin{array}{c}\text { ATG TGC AGY ACC AGT AAR } \\
\text { GTK ATG GC } \\
\text { TGG GTR AAR TAR GTS ACC } \\
\text { AGA AYC AGC GG }\end{array}$ & 593 bp & $\begin{array}{c}\text { Archambault } \\
\text { et al., } \\
2006\end{array}$ \\
\hline
\end{tabular}

\subsection{Incidence of $E$, coli infection in chicken}

Out of 300 recently dead broilers from commercial farm were subjected to postmortem examination to detect birds that show pericarditis, perihepatitis and airsacculitis (72) different bacterial isolates of Enterobacteriaceae suspected to be $E$. coli which identified as (56)fifty six E. coli,(1) one shigella,(1) one Enterobacter and (14) fourteen kluyvera ( Table 2 \&figure (1)

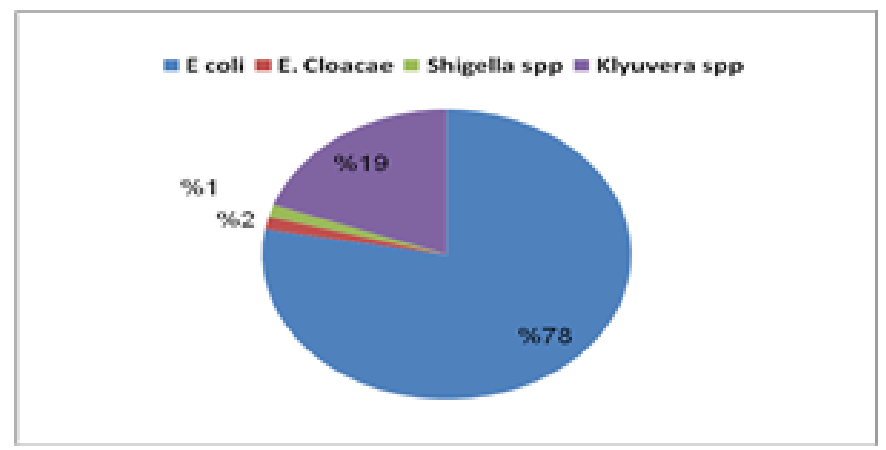

Fig. 1. Total bacteriological isolates of samples 
3.2. Serogrouping of E, coli isolates recovered from chicken samples

The most commonly E. coli isolated were 4 (O125), 3(O158) ,2( O55), 2(O129) ,1(O27), 1(O115), 2(O8), 1(O142), 2(O20) ,2(O6) and 4 untyped (Table 3 ) .

\subsection{Virulence test results:}

\subsubsection{Conge red binding ability}

Congo red binding ability test were revealed that all of $56 \mathrm{E}$ coli isolates showed Congo red binding activity giving red colonies and considered Congo red positive $(\mathrm{CR}+)$.

\subsubsection{Hemolytic activity on Blood A gar}

Out of $56 \mathrm{E}$. coli isolates, only one isolate gave beta hemolysis

\subsection{Antibiogram sensitivity test}

\subsection{Antibioticresistance genes detection of $\mathrm{E}$, coli:}

Twelve sero groups isolates of E. coli were differed about prescence or absence of resistance gene as mentioned in table 5 . fig (2), (3) \&(4) using the specific primers for Aada2 , BlaCTX and $\operatorname{Tet} \mathrm{A}(\mathrm{A})$ genes respectively.

\section{Table (4): Antibiogram result of E coli serogroupes}

\begin{tabular}{|c|c|c|c|c|c|c|c|c|c|c|c|c|}
\hline \multirow{2}{*}{$\begin{array}{c}\text { E. coli } \\
\text { serotype } \\
s\end{array}$} & \multicolumn{12}{|c|}{ Antibiotics } \\
\hline & CIP 5 & $A \times 25$ & CTX30 & NOR10 & OFXS & DA2 & CT10 & SXT25 & E15 & DO30 & ENR & $\mathrm{S}$ \\
\hline 020 & $\mathbf{R}$ & $\mathbf{R}$ & $\mathbf{R}$ & $\mathbf{R}$ & $\mathbf{R}$ & $\mathbf{R}$ & $\mathrm{s}$ & $\mathbf{R}$ & $\mathbf{R}$ & $S$ & $S$ & $\mathbf{R}$ \\
\hline 020 & $\mathrm{~S}$ & $\mathbf{R}$ & $\mathbf{R}$ & 5 & 5 & $\mathbf{R}$ & $\mathrm{S}$ & $\mathbf{R}$ & $\mathbf{R}$ & $\mathbf{R}$ & 5 & $\mathbf{R}$ \\
\hline 0125 & 5 & $\mathbf{R}$ & 1 & $\mathbf{R}$ & 1 & $\mathbf{R}$ & 5 & $\mathbf{R}$ & $\mathbf{R}$ & $\mathbf{R}$ & 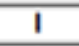 & $\mathbf{R}$ \\
\hline 0125 & 1 & $\mathbf{R}$ & $\mathbf{R}$ & $\mathbf{R}$ & 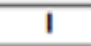 & $\mathbf{R}$ & $\mathrm{S}$ & $\mathbf{R}$ & $\mathbf{R}$ & $\mathbf{R}$ & $\mathbf{I}$ & $\mathbf{R}$ \\
\hline 055 & $\mathbf{R}$ & $\mathbf{R}$ & $\mathbf{R}$ & $\mathbf{R}$ & $\mathbf{R}$ & $\mathbf{R}$ & 5 & 5 & $\mathbf{R}$ & 1 & $\mathbf{R}$ & $\mathbf{R}$ \\
\hline 055 & $\mathbf{R}$ & $\mathbf{R}$ & $\mathbf{R}$ & $\mathbf{R}$ & $\mathbf{R}$ & $\mathbf{R}$ & $\mathrm{S}$ & 5 & $\mathbf{R}$ & $I$ & $\mathbf{R}$ & $\mathbf{R}$ \\
\hline 0115 & $\mathbf{R}$ & $\mathbf{R}$ & $\mathbf{R}$ & $\mathbf{R}$ & $\mathbf{R}$ & $\mathbf{R}$ & $\mathbf{R}$ & $\mathbf{R}$ & $\mathbf{R}$ & $\mathbf{R}$ & $\mathbf{R}$ & $\mathbf{R}$ \\
\hline 0142 & $\mathbf{R}$ & $\mathbf{R}$ & $\mathbf{R}$ & $\mathbf{R}$ & $\mathbf{R}$ & $\mathbf{R}$ & $\mathbf{R}$ & $\mathbf{S}$ & $\mathbf{R}$ & $S$ & $\mathbf{R}$ & $\mathbf{R}$ \\
\hline 0142 & $\mathbf{R}$ & $\mathbf{R}$ & $\mathbf{R}$ & $\mathbf{R}$ & $\mathbf{R}$ & $\mathbf{R}$ & $\mathbf{R}$ & 5 & $\mathbf{R}$ & 5 & $\mathbf{R}$ & $\mathbf{R}$ \\
\hline 06 & $\mathrm{~S}$ & $\mathbf{R}$ & $\mathbf{R}$ & 1 & 5 & $\mathbf{R}$ & $\mathbf{R}$ & $\mathbf{R}$ & $\mathbf{R}$ & 1 & $\mathbf{R}$ & $\mathbf{R}$ \\
\hline 08 & $\mathrm{~S}$ & $\mathbf{R}$ & $\mathbf{R}$ & 1 & 1 & 1 & $\mathbf{R}$ & $\mathbf{R}$ & $\mathbf{R}$ & $\mathbf{R}$ & $\mathbf{R}$ & $\mathbf{R}$ \\
\hline 08 & $\mathbf{R}$ & $\mathbf{R}$ & $\mathbf{R}$ & 1 & $\mathbf{R}$ & $\mathbf{R}$ & $\mathbf{R}$ & $\mathbf{R}$ & $\mathbf{R}$ & $\mathbf{R}$ & $\mathbf{R}$ & $\mathbf{R}$ \\
\hline 0158 & $\mathbf{R}$ & $\mathbf{R}$ & 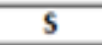 & 1 & $\mathbf{R}$ & $\mathbf{R}$ & $\mathbf{R}$ & $\mathbf{R}$ & $\mathbf{R}$ & $\mathbf{R}$ & $\mathbf{R}$ & $\mathbf{R}$ \\
\hline 0158 & $\mathbf{R}$ & $\mathbf{R}$ & 5 & 1 & $\mathbf{R}$ & $\mathbf{R}$ & $\mathbf{R}$ & $\mathbf{R}$ & $\mathbf{R}$ & $\mathbf{R}$ & $\mathbf{R}$ & $\mathbf{R}$ \\
\hline 0158 & 5 & $\mathbf{R}$ & 5 & $\mathbf{R}$ & $\mathbf{R}$ & $\mathbf{R}$ & $\mathrm{S}$ & $\mathrm{I}$ & $\mathbf{R}$ & $\mathbf{R}$ & $\mathbf{R}$ & $\mathbf{R}$ \\
\hline 027 & $\mathbf{R}$ & $\mathbf{R}$ & $\mathbf{R}$ & $\mathrm{S}$ & $\mathbf{R}$ & $\mathbf{R}$ & $\mathbf{R}$ & $\mathbf{R}$ & $\mathbf{R}$ & $\mathbf{R}$ & $\mathbf{R}$ & $\mathbf{R}$ \\
\hline 027 & $\mathbf{R}$ & $\mathbf{R}$ & $\mathbf{R}$ & $S$ & $\mathbf{R}$ & $\mathbf{R}$ & $\mathbf{R}$ & $\mathbf{R}$ & $\mathbf{R}$ & $\mathbf{R}$ & $\mathbf{R}$ & $\mathbf{R}$ \\
\hline 0129 & $\mathbf{R}$ & $\mathbf{R}$ & $S$ & $\mathbf{R}$ & $\mathbf{R}$ & $\mathbf{R}$ & $S$ & 1 & $\mathbf{R}$ & $\mathbf{R}$ & $\mathbf{R}$ & $\mathbf{R}$ \\
\hline 0125 & $\mathbf{R}$ & $\mathbf{R}$ & $\mathbf{R}$ & $\mathbf{R}$ & $\mathbf{R}$ & $\mathbf{R}$ & $\mathbf{R}$ & $\mathbf{R}$ & $\mathbf{R}$ & $\mathbf{R}$ & $\mathbf{R}$ & $\mathbf{R}$ \\
\hline 0125 & $\mathbf{R}$ & $\mathbf{R}$ & $\mathbf{R}$ & $\mathbf{R}$ & $\mathbf{R}$ & $\mathbf{R}$ & $\mathrm{S}$ & $\mathbf{R}$ & $\mathbf{R}$ & $\mathbf{R}$ & $\mathbf{R}$ & $\mathbf{R}$ \\
\hline
\end{tabular}


Table (5): PCR analysis for antibiotic resistance genes (aaDA2 - blaCTX - tetA(A)

S: sensitive $\quad \mathrm{R}$ : resist

\begin{tabular}{|c|c|c|c|c|}
\hline \multirow{2}{*}{$\begin{array}{l}\text { ID of } \\
\text { the } \\
\text { samples }\end{array}$} & \multirow{2}{*}{$\begin{array}{c}\text { E. coli } \\
\text { serotypes }\end{array}$} & \multicolumn{3}{|c|}{ Results } \\
\hline & & Aada 2 & BlaCTX & $\operatorname{TetA}(A)$ \\
\hline 1 & O20 & Positive @ & Positive ${ }^{\circledR}$ & Positive ${ }^{\circledR}$ \\
\hline 2 & 0125 & Positive ® & Negative(S) & Positive ${ }^{\circledR}$ \\
\hline 3 & O20 & Positive® & Positive ® & Positive ${ }^{\circledR}$ \\
\hline 4 & 0125 & Positive® & Positive ® & Positive $\AA$ \\
\hline 5 & 055 & $\begin{array}{l}\text { Negative } \\
\text { (S) }\end{array}$ & Positive ${ }^{\circledR}$ & Positive ${ }^{\circledR}$ \\
\hline 6 & 055 & Negative(S) & Positive $®$ & Positive $®$ \\
\hline 7 & 0158 & Negative(S) & Positive ${ }^{\circledR}$ & Positive ${ }^{\circledR}$ \\
\hline 8 & 0158 & Negative(S) & Negative(S) & Positive ${ }^{\circledR}$ \\
\hline 9 & 0158 & Positive ${ }^{\circledR}$ & Negative(S) & Positive ${ }^{\circledR}$ \\
\hline 10 & $\mathbf{0 2 7}$ & Negative(S) & Negative(S) & Positive ${ }^{\circledR}$ \\
\hline 11 & $\mathbf{O 2 7}$ & Positive ${ }^{\circledR}$ & Negative(S) & Positive ${ }^{\circledR}$ \\
\hline 12 & 0129 & Positive ${ }^{\circledR}$ & Negative(S) & Positive ${ }^{\circledR}$ \\
\hline
\end{tabular}

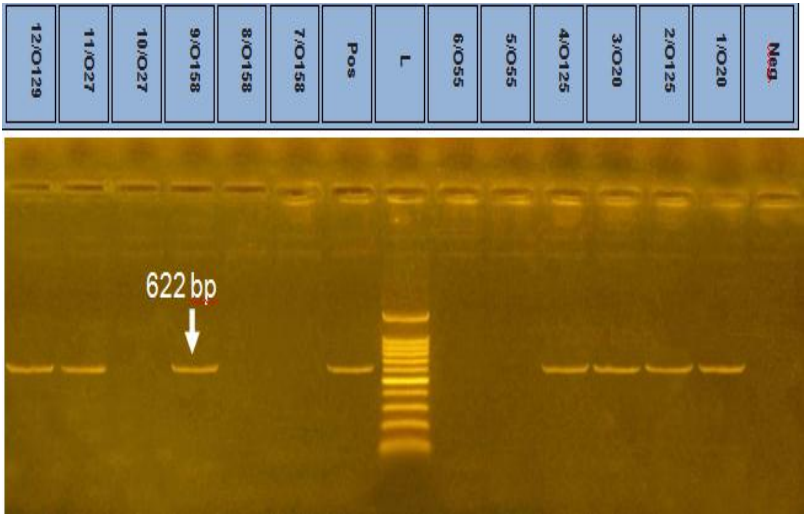

Fig. 2. Electrphoretic pattern of PCR products (622 bp) specific for aada 2 gene of $E$. coli in agarose gel stained with ethidium bromide

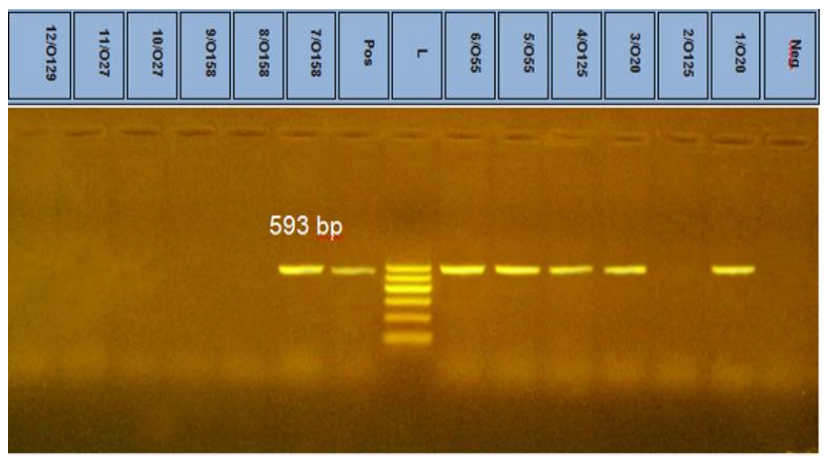

Fig. 3. Electrphoretic pattern of PCR products (593 bp) specific for blaCTX gene of E. coli in agarose gel stained with ethidium bromide.

\section{Discussion}

In this study, E. coli was detected after pre-enrichment on BPW. Then inoculated direct on agar medium (MacConky agar, VRBL and EMB agar), Typical colonies on TSA were used for further morphological and biochemical identification The typical E. coli colonies were typing by antisera. In the present work, all E. coli strains showed lactose fermentation (pink colonies) on MacConkey agar and green metallic sheen colonies on EMB .Out of 300 recently dead broilers from commercial farms were (72) different bacterial isolates of Enterobacteriaceae suspected $E$. coli were identified as (56) fifty six E. coli,(1) one shigella,(1) one Enterobacter spp and (14) fourteen kluyvera spp. 
Congo red binding ability test revealed that all of $56 \mathrm{E}$ coli isolates showed Congo red binding activity giving red colonies and considered Congo red positive $(\mathrm{CR}+)$. The degree of redness of the colonies varied from one isolate to another. Only The strains which gave more powerful results (dark red colony) are selected for sero grouping The results were in agreement with Berkhoff and Vinal [1986], who reported a strong correlation between expression of $\mathrm{CR}$ phenotype and virulence in avian E. coli. Pathogenic E. coli can be identified by their ability to bind $\mathrm{CR}$ and produce brick red colonies .

The most prevalent serogroups were $4(\mathrm{O} 125), 3(\mathrm{O} 158), 2($ O55) , 2(O129) ,1(O27), 1(O115), 2(O8), 1(O142), 2(O20) ,2(O6) and 4 untyped (table ,4) A wide variety of E. coli serogroupes and non-subtypes from broiler in Egypt were also reported Taha et al 2002.

Antibiogram test was $100 \%$ to AX, DA, E and S; $85,7 \%$ to DO ; $80 \%$ CTX , $76 \%$ to OFX and CIP; $61.8 \%$ to CT .

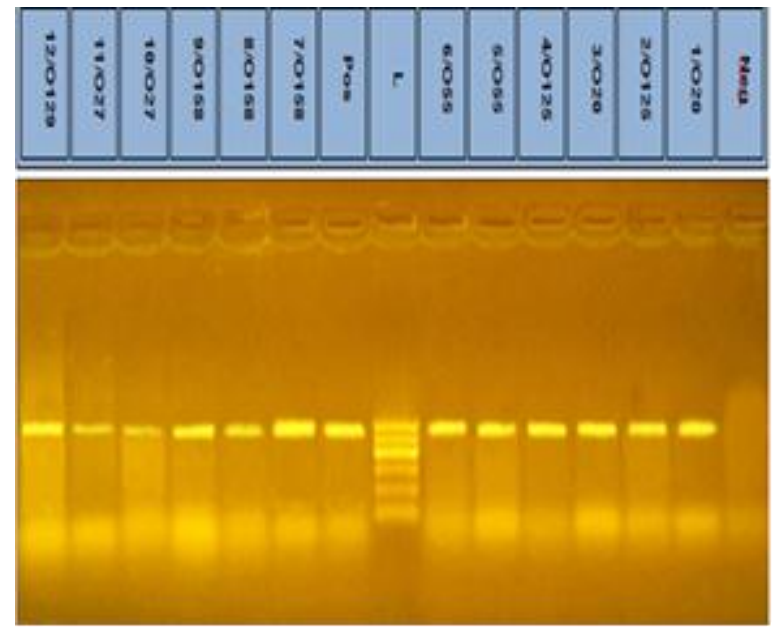

Fig. 4. Electrphoretic pattern of PCR products ( bp) specific for tet $A$ gene of $E$, coli in agarose gel stained with ethidium bromide.

SXT and $57 \%$ to Nor. Similar results were reported by Ozawa et al [2008]. The present study detected most of isolates was resistance to doxycycline with percent $85.7 \%$ by disc diffusion which agree with 'Moon et al (2011) that studied the actual frequency of antimicrobial resistance in fecal Escherichia coli isolated from .One hundred and nine E.coli isolates were higher resistant to ampicillin (68.8\%) streptomycin (60.6\%), ciprofloxacin (65.1\%), and tetracycline $(96.3 \%)$.
PCR analysis for antibiotic resistance genes of E. coli Twelve sero groups isolates of $\mathrm{E}$. coli were differed on result using the specific primers for aaDa2, blaCTX and tetA(A) genes respectively. The obtained results were recorded (Table 5) .

\section{References}

Archambault, M.; Petrov, P.; Hendriksen, R.S.; Asseva, G.; Bangtrakulnonth, A.; Hasman, H.; Aarestrup, F.M. (2006). Molecular characterization and occurrence of extended-spectrum beta-lactamase resistance genes among Salmonella enterica serovar Corvallis from Thailand, Bulgaria, and Denmark. Microb Drug Resist. 2006 Fall;12(3):192-8.

Bandyopadhay, P.K., Dhawedkar, R.G. (1984). E.coli salpingoperitonitis in poultry. Indian Vet. J., 61:348-349.

Berkhoff, H. A. and Vinal, A. C. (1986): Congo red medium to distinguish between invasive and non-invasive Escherichia coli pathogenic for poultry. Avian Dis., 30(1): 117-121.

Cheesbrough, M., (2000). District Laboratory Practice in Tropical Countries.1st Ed., Press Syndicate of the University of Cambridge, Cambridge UK., 132-143.

Cruickshank, H., Duguid, J.P., Marmon, B.P., Swain, R.H.A. (1975). Medical Microbiology. The practice of Medical Microbiology, 12th Ed. Churchill Livingstone, Edinburgh. London and New York.

El-Jakee J.K, R.M. Mahmoud, A.A. Samy, Mona A. ElShabrawy, M.M. Effat and W.A. Gad El-Said Molecular Characterization of E. coli Isolated from Chicken, Cattle and Buffaloes International Journal of Microbiological Research 3 (1): 64-74, 2012

Koneman, E. W., Allen, S. D., Janda, W. M., Schreckenberger, P. C. and Winn, W. C., (1992). Diagnostic Microbiology. 4th Ed. Philadelphia. New York.

Marilda, C., Ernest, E., Julio, C., Amauri, A., Lvens, G. And Diogenes, S. (1990). Virulence factors of avian Escherichia coli. Avian Dis., 34, 531-538.

Mary, C. and Usha, M. (2013). Incidences of multi-drug resistance Escherichia coli isolates in Panipuri sold in Bangalore. Inter. Food Res. J., 20 (2): 1007-1009.

Moawad, A.A., Hotzel, H., Awad, O., Tomaso, H., Neubauer, H., Hafez, H.M. and ElAdawy, H. (2017). 
Occurrence of Salmonella enterica and w in raw chicken and beef meat in Northern Egypt and dissemination of their antibiotic resistance markers. Gut Pathog., 9: 57.

'Moon Ho Jang, 'Jae Keun Cho, iDong MiKwak, 'Gil-Jae Cho, Young Ju Lee (2011). Antimicrobial Resistance and Resistance Gene Determinants of Fecal Escherichia coli Isolated from Chicken. Korea Journal of Animal and Veterinary Advances 10(24): 3308-3311.

Nawaz, M. Abbas Irshad, Mubarak Ali* and Ahsan-ulHaq (2016). Effect of probiotics on growth performance , nutrient digestability and carcass characteristics in broilers The Journal of Animal \& Plant Sciences, 26(3): 2016, Page: 599-604.

NCCLS "National Committee for Clinical Laboratory Standards" (2001). Performance standards for antimicrobial susceptibility testing. Supplement M100S11. Villanova, PA, USA

Ozawa, M., Harada, K., Kojima, A., Asi, T. and Sameshima, T. (2008). Antimicrobial susceptibilities, sero $\neg$ groups, and molecular characterization of avian pathogenic Escherichia coli isolates in Japan. Avian Dis., 52: 392-397.

Quinn, P.J., Markey, B.K., Carter, M.E., Donnelly, W.J.C., Leonard, F.C. (2002). Veterinary microbiology and microbial diseases.1st Iowa State University Press Blackwell Science.

Randall, L.P.; Cooles, S.W.; Osborn, M.K.; Piddock, L.J.V. and Woodward, M.J. (2004). Antibiotic resistance genes, integrons and multiple antibiotic resistance in thirty-five serotypes of Salmonella enterica isolated from humans and animals in the UK. Journal of Antimicrobial Chemotherapy. 53, 208-216.
Salama, S. S, Afaf A. K., Elham A. E. and Taha, M. M. (2007). Molecular Strategies for the differentiation and identification of local E. coli isolated from chicken I. characterization of protein profile. B.S. Vet. Med. J. 17 (1) 25-28.

Salyers, A.A., Gupta, A. and Wang, Y. (2004). Human intes $\neg$ tinal bacteria as reservoirs for antibiotic resistance genes. Trends Microbiol., 12(9): 412-416.

Hering J1, Frömke C1, von Münchhausen C1, Hartmann M1, Schneider B1, Friese A2, Rösler U2, Kreienbrock L1, Hille K3 Cefotaxime-resistant Escherichia coli in broiler farms-A cross-sectional investigation in Germany. Prev Vet Med. 2016 Mar 1;125:154-7. doi: 10.1016/j.prevetmed.2016.01.003.

Taha, M., Ibrahim, R.S. and Hussien, A.A.A. (2002). Studying the pathogenicity and RAPD PCR analysis of dif $\neg$ ferent Escherichia coli serotypes isolated from broilers and layer chickens. Assiut Vet. Med. J., 46(92): 224-236.

Vandekerchove, D., De Herdt, P., Leavens, H. and Pasmans F. (2004 a). Colibacillosis in caged layer hens characteristics of the disease and the aetiological agent. Avian Pathol., 33(2) 117-/125.

Walker, R. A., Lindsay, E., Woodward, M. J. et al. (2001). Variation in clonality and antibiotic-resistance genes among multi-resistant Salmonella enterica serotype Typhimurium phage-type U302 (MR U302) from humans, animals, and foods. Microbiological Research 7, 13-21.

Yousef, S.A., Ammar, A.M. and Ahmed, D.A. (2013). Serological and molecular typing of avian pathogenic E. coli originating from outbreaks of colibacillosis in chicken flocks. Internat. J. Sci. Res., 4(2): 2082-2088 